\title{
A Hybrid Instructional Model for Post Graduate Education: A Case Study from the United Arab Emirates
}

\author{
Patrick Dougherty \\ Abu Dhabi Men’s College
}

\author{
Josephine Butler \\ Abu Dhabi Women's College
}

\author{
Sarah Hyde \\ Dubai Women's College
}

\begin{abstract}
This case study analyzed the benefits and drawbacks of an experimental multi-site, hybrid, or blended, instructional delivery system for post-graduate education in the United Arab Emirates. Two courses as part of a Masters in Educational Studies (MEST) program were taught simultaneously from two locations. The classes were linked electronically via a dual screen system that allowed class members to see one another and lecture materials in real time. The instructor was physically present at each site on a rotating basis, and was supported by location designated tutors who remain at each site. This method of delivery was intended to allow students in remote locations to participate in degree programs, increase the offerings available, and utilize the full potential and expertise of the teaching staff.

There were two research questions guiding the study: (1) Did students perceive the hybrid delivery system as beneficial to their learning objectives, and (2) according to the students, what were the benefits and drawbacks of the delivery system? The data indicated general student satisfaction with the hybrid delivery system and identified several benefits of, and drawbacks to, the delivery structure.
\end{abstract}

\section{Introduction}

This case study analyzed the benefits and drawbacks of a multi-site, hybrid, or blended delivery system for post-graduate education in the United Arab Emirates. The guiding research questions were: (1) Did students perceive the hybrid delivery system as beneficial to their learning objectives, and (2) according to the students, what were the benefits and drawbacks of the delivery system?

Two courses as part of a Masters of Educational Studies program (MEST) were taught simultaneously from two locations, one a college in Dubai, and the other a college in Abu Dhabi. The classes were linked electronically via a dual screen system that allowed class members to see one another and lecture materials in real time. Class presentations, lectures, discussions, and videos, were shared in real-time electronically.

The instructor was physically present at each site on a rotating basis, and was supported by location designated tutors, with one tutor for each location. In addition to the tutors, each location had information technology (IT) staff in attendance during class sessions. The physical environment for both classes, and at both sites, was in conference room facilities and not in traditional classrooms. The room at the Dubai location had a conference table and several extra desks and the Abu Dhabi site had a conference table as well as desks. Each class met six times, on alternating Saturdays, with separate times to meet with the tutors. Each class meeting was five hours in length. Students were expected to write article critiques, research papers, and conduct team presentations on education related topics. The calendar length of each course was approximately fifteen weeks including research time.

The study was conducted over the course of two graduate courses and involved thirty-two students. There were thirty women and two men ranging in ages from the mid-20s to approximately sixty years of age. The majority of them were United Arab Emirates (UAE) nationals, with the remainder being from other countries in the Middle East. Most were educators at the primary or secondary levels with a few working either for government ministries or local universities. All were able to enter the English language Masters of Educational Studies program which indicated that their level of English was at least of a band 6.5 of the International English Language Testing System (IELTS) test. Anecdotally, all were capable of fully participating in five hour classes conducted in English and all were able to write required papers of between 1000 and 4000 words in English as part of their courses. This was significant as all of the research tools utilized English as the medium of data collection. The data itself was collected via an internet based survey, a panel discussion held at 
a regional teacher education conference, and three focus groups.

The internet-based survey was voluntary, anonymous, and responses were collected from approximately $31 \%$ of the possible respondents. The panel discussion had seven panelists, all members of either the Dubai or Abu Dhabi cadres of the MEST program. The members of the panel were volunteers. A recording of the discussion was made and transcribed for the sake of the case study. The three focus groups were made up of nineteen MEST students in total. Those who had volunteered to be on the panel discussion were excluded from volunteering for the focus groups. The moderators of the focus groups made recordings of the respondents' answers and ensuing discussions. These recordings were later transcribed for use in this study. Between the panel discussion and focus groups, $81 \%$ of the class offered their perspectives on the research questions. Their perceptions of the efficacy of the delivery system formed the core of the research data.

\section{Research Rationale}

The two courses taught in the MEST program involved a hybridization of instructional delivery. It was a blending of videoconferencing, face-to-face instruction, the presence of a site-dedicated tutor, and the use of e-mail for assignment submission, clarifying questions, and support. The growing importance of videoconferencing was highlighted in an interview with Bill Gates on BBC's Hardtalk [8] "The idea of videoconferencing is going to get so much better than it is today." Lawson et al. [13] describes videoconferencing as a means to increase student intake and form links between geographically distant institutions. He sees it as a cost effective "lecture at a distance" reaching out to many while at the same time avoiding duplication. However, there was little interest in the early stages in analyzing and enhancing the pedagogy used.

The blended delivery system was intended to allow students in remote locations to participate in degree programs, increase the offerings available, and utilize the full potential and expertise of the teaching staff. But it was experimental and the researchers decided that research into the perceived efficacy of the delivery model was important in that the institution sponsoring the program was contemplating utilizing this delivery format for its entire MEST program. Before that was done, the researchers, who were the instructor and tutors for the courses, felt that an examination of the specific benefits and drawbacks of this delivery system, with its mix of technology and "old school" methods (digital connections, but with the presence of the instructor on alternate classes and the permanent placement of a tutor dedicated to the support of a cadre of students) was warranted. The results were designed to inform local policy makers, but the issue of providing educational opportunities to remote sites, the best use of technology and staff, and what educational formats are perceived by students as supportive of their educational goals are useful for policy makers and educators in general.

Utilizing technology for course delivery has become popular over the past decade with many institutions offering on-line and post-graduate degrees. For many students it has offered the opportunity to improve their skill sets, obtain certifications and degrees, and continue to honor the social or familial commitments that might have kept them from pursuing educational opportunities in the past. However, some learners require more than just an on-line program to fulfill their academic needs. They might also require the presence of the instructor and a support network that is found in traditional school settings. Hence, the need for blended learning formats that mixed the technological with the human, and provided the benefits of both systems of delivery.

In its most basic definition blended learning is a fusion of face-to-face and on-line learning. As Dziuban, Hartman, and Moskal [5] noted, blended learning should be understood as an instructional approach that unites the socialization of the traditional classroom environment with the possibilities and active learning aspects of the on-line educational medium. There has been significant research into videoconferencing, much of it positive. Zhao and Angelova see it as very similar to face-to-face communication. In terms of teleeducation in the medical field O'Regan et al. evaluated the effectiveness of videoconferencing in the delivery of a radiology course to students in distant locations. Overall satisfaction was high. In the case of language learners, Yamada and Akahori (in Zhao and Angelova) found that communicative language learning works well in videoconferencing "as it provokes a large amount of input and many opportunities for meaning negotiation” (p. 12). Gill et al. [10] reflected on their experiences of using videoconferencing for a number of years and offer advice for current users of this technology. They believe that when well planned, and delivered effectively, videoconferencing 
teaching sessions can be stimulating and enjoyable.

For institutions Andrews describes the advantages for students, satellite campuses, and staff. Students can have access to expertise despite location. Instructors from satellite campuses can take the lead in the teaching rather than the central campus. This gives their staffs, who often feel at a disadvantage, more opportunities. Campuses can work together to offer a whole course, thus increasing course offerings. The collaboration and knowledge sharing required for these developments are a very important professional development activity.

Dziuban, Hartman, and Moskal [5] also found that blended courses could reduce student drop-out rates and improve student learning more so than in purely online courses. GeorgeWalker \& Keeffe [9] suggested that the mix of blended learning and information and communication technologies can create circumstances where students increase their engagement with the content and meet their academic and personal goals.

Gillies [11] reported that students felt that they had more opportunities to ask questions of the instructor, and that there was more group interaction within the class than there would be in a regular university lecture hall. They also felt that the instruction was more personal. This would also tie-in with Gilles observation that some students who, for personal reasons, would not be able to give-up a year away from home and stay on a campus benefit from the on-line and video conferencing elements of blended learning. It was also reported in Gillies [11] that students developed a sense of togetherness, camaraderie, and shared experience that came about due to their geographical isolation. EriksBrophy et al. [6] described the cost effectiveness of videoconferencing, as well as the reduced financial costs associated with travel, accommodation and time away from work. The University of Exeter reported that 460 videoconferencing sessions saved approximately 96,600 miles of staff travel; 63 weeks of staff time and $\mathrm{CO} 2$ emissions reduced by 34 tonnes. However, there are drawbacks to blended learning.

Technological failure can be a distraction, and effective technological ties between sites are imperative (Exeter, U., [7]). Gill, Parker, and Richardson [10] and Andrews and Klease [2] cited the necessity for a team approach and suggest that such a team might be made up of a lead teacher who is responsible for content delivery, supported by site designated tutors and constant technological support at each instructional location when videoconferencing is used. Having a tutor present at each site was seen as to be desirable as students valued the "off-air" times as an opportunity to seek clarifications and generally interact with the tutors (Gillies, [11]). Gill, Parker, and Richardson [10] stressed the importance of a good working relationship with technical support for effective video conferencing delivery.

Luck [14] conservatively estimates that twothirds of the material covered in the standard classroom may be delivered in a videoconference session. Thus, any blended learning program that involved videoconferencing would have to be realistic and perhaps creative in delivery design. Hofmann in George Walker et al. [9] saw skilled facilitation and ongoing support for learners as crucial in any blended delivery program.

According to Lawson et al. [13] other challenges to providing a successful blended delivery program involve the possible distraction of students at the remote sites, with increased off-topic chatting and less interaction between the students and the instructor. Issues of self management become important when the instructor is not physically present with the students.

Blended learning courses promote learner autonomy (Motteram, [15]). However, it must also be noted that a badly designed blended learning program can decrease effective learning compared to a single delivery method.

Andrews inventories the applications videoconferencing can offer to provide a very rich and interactive learning environment, one in which students enjoy and find more preferable than the "talking head presentation". Therefore, it is not surprising that many researchers (Andrews \& Klease [1]) recommend strategic staff development to improve familiarity with, understanding of this medium of delivery and thus be best equipped to exploit what videoconferencing has to offer. As one of the subjects of this study explained, "For me I feel whether the instructor is here or in Dubai the environment makes us feel that we are in one place” (Butler, Hyde, Dougherty, [3]).

\section{Contribution to Knowledge}

The research questions were (1) did students perceive the blended delivery system as beneficial to their learning objectives, and (2) according to the students, what were the benefits and drawbacks of the delivery system? Regarding the first question, of whether or not the students perceived the blended learning 
format to be beneficial, the overall findings indicated that the system was beneficial. We can examine specific elements of this response by looking at the benefits of the blended learning format as illuminated by the students. Their comments fell into three broad categories: discussing the video conferencing, the face to face communication, and the support provided by the tutors.

In the case of video conferencing, $100 \%$ of the survey respondents felt that this was a positive experience. Comments were offered to back up this response.

One of the survey respondents stated that the experience was, "Exciting, useful and attractive" and that he or she did not ". . . feel the physical absence of the lecturer because with videoconference I could see him and carry out the discussion face to face via the screen" (Dougherty, Butler, Hyde, [4]). Another subject, when asked about the differences between the having the instructor physically present in the room and the video conference experience had this to say, "It felt the same!" The same subject later explained that this also might have been due to having “. . . tutors . . . in both campuses, which made questioning and moving the flow of classes easy". Still another respondent explained that there was "No big difference as long as we can communicate. It was [the] same experience as face to face". Another subject added that, “. . . it is practical, interesting, modern and meaningful - I like it!” (Dougherty, Butler, Hyde, [4]). Finally, one subject offered that, "It was quite [an] interesting experience; it felt as if we were in a normal classroom environment".

Focus group members found video conferencing was particularly beneficial in terms of communication and engagement. One subject had this to say regarding the experience (Butler, Hyde, Dougherty, [3]): “. . . video links are very good. Students can still be with the professor even if he is in another city, giving the chance to all students to attend the lectures. It is so brilliant an idea that I can't think of drawbacks."

Another subject felt the connection that can be developed by students from different regions was one of the positive aspects of the experience, as he or she explained, “. . . it works as a great assistance in putting together students regardless of their geographical region[s]" (Dougherty, Butler, Hyde, 2010).

In the case of face to face communication, students highlighted that they benefited from interacting with their fellow students and learning about their viewpoints as members of communities in other parts of the country. They believed that video conferencing aided greater participation rates, more interactions, and provided an opportunity to meet each other and share experiences. Indeed, in the panel discussion panelists commented on how they felt they knew each other and this despite the panel discussion being the first time that they had physically met. As one respondent put it, "The face-to-face communications with professors and peers on-site and via video link enhances greater interactions and exchanges of ideas; it is always meaningful for me to meet with classmates” (Hyde, Butler, Dougherty, [12]).

While students were positive about viewing and sharing ideas with one another via the video conferencing, they did feel it was important for the lecturer to alternate his physical presence between Abu Dhabi and Dubai campuses. As one focus group member explained, “. . . it was good that Dr. Pat was moving in between, rather than staying in one place. Otherwise we will feel really missed or out of the picture" (Butler, Hyde, Dougherty, [3]). A survey respondent mentioned that "By being physically present, I believe that it gives both the student and lecturer a better understanding of each other. Also, sometime some questions need to be addressed in person [and] that is when the physical presence makes a difference" (Dougherty, Butler, Hyde, [4]).

The multi-site delivery system was a benefit according to the data. Many of the students work full time and have busy family lives; the flexibility offered by the multi-site delivery mode was seen as time saving in terms of travel and allowed students the opportunity to attend sessions at either the Dubai or Abu Dhabi campus. We learned from the survey respondents that $60 \%$ of them would not have been willing to travel extensively to attend classes (Dougherty, Butler, Hyde, [4]). As one focus group member stated, "For me, I don't have to go to Abu Dhabi if I'm closer to Dubai; so, it's much easier for us and it is working" (Butler, Hyde, Dougherty, [3]).

Tutors were viewed as invaluable by all the respondents in the survey, the focus groups, and by the panel discussion members. Many felt the tutor's presence at all the sessions was necessary, in particular as the lecturer alternated between sites. Members of the focus groups commented how they found the tutor useful as a resource for clarification or to give an opinion and for general support. One student commented that knowing the tutor was there made her feel more secure. The focus group discussions highlighted the importance of the tutors, especially at the beginning of the MEST program. Some students had not studied recently and required intensive support with 
English, time management, and study skills, including referencing (Butler, Hyde, Dougherty, [3]).

Regarding the issue of technology, the participants commented on how smoothly the video conferencing ran and the lack of serious technological glitches. They felt that the quality of connection aided effective communication. As respondent pointed out, the system was “. . . very high tech, and I didn't feel disconnected from the instructor. It moves very smoothly, the communication was clear" (Hyde, Butler, Dougherty, [12]).

The second part of the second research question required the panelists, focus group members, and survey respondents to identify the drawbacks to the blended delivery system. These were few in number and focused on one physical issue with the structure of a conference room, and on an issue of human nature.

There were some complaints about noise interference in that the microphone at the Dubai location was placed on the conference table which students sat around and it was able to pick up any movement of papers, books, or bags that were placed on the table. As the microphone was built into the conference table, it could not be moved. Occasionally, if there was a presentation being done in Abu Dhabi, the tutor would mute the microphone to keep from distracting the presenter(s).

Additionally, two of the survey respondents felt that the class was less disciplined when the lecturer was at the other location and they were participating via the video link. As one respondent explained (Dougherty, Butler, Hyde, [4]), "I think the lecturer has more control over the class when he is physically there than when he is giving the lecture thorough videoconference." Another subject explained that, "Students, when alone with a camera and no lecturer are more lax on class rules, talk more and are less attentive".

Another issue was that students felt that they were more engaged and asked more questions when the lecturer was physically present rather than when his input was viewed via video conference. Participants in the focus groups noted that they were quieter and interacted less when the lecturer was at the other location. As one of the respondents explained, “. . . when Dr. Pat is here, we participate more, but when he's there we hesitate to answer direct questions” (Butler, Hyde, Dougherty, [3]).

\section{Conclusion}

This case study was conducted at two colleges in the United Arab Emirates and evaluated the efficacy of a hybrid delivery system that utilized video conferencing, the lecturer's presence on-site during alternating class meetings, and the presence of tutors dedicated to the continual assistance of one of the cadres. From their responses in the survey, in the focus groups, and from the panel discussion, the students answered the guiding questions of this study.

Did students perceive the blended delivery system as beneficial to their learning objectives? Yes, their responses indicated that they felt the blended delivery system met their needs and aided them in their learning.

According to the students what were the benefits and drawbacks of the delivery system? In their responses there were more benefits listed than drawbacks. The benefits were multiple, everything from allowing connections with other students to making the course, and access to the location where it was being offered, more convenient. Also, they indicated that the drawbacks were rooted in easily overcome technical issues, such as the placement of a microphone, or the greater issue of human nature, that being that when the instructor was at the other site students would not be as attentive or participate as much. This indicates the need for a careful analysis of the structure of the video conference classroom, insuring that equipment be placed appropriately and in such a way as to minimize distraction. Also, the possibility of class inattention might be ameliorated by increasing the role of the tutor in the class management when the instructor is at the alternate location.

This study has led the researchers to ask additional questions, to be tackled in future research projects. Further research needs to be done to identify what the best pedagogical design is for a hybrid delivery system and to find the best classroom management system for such a design. Additionally the researcher would like to conduct a longitudinal comparison study between a traditional class structure, completely on-line formats of instruction, and the hybrid delivery paradigm. The hope is to determine the relative strength of the hybrid delivery system in comparison to the other two instructional formats. It is hoped that such a comparison study might illuminate why one survey respondent (Dougherty, Butler, Hyde, [4]) was wont to write that the hybrid delivery system, with its mixture of old school and new school design was, "More engaging than the traditional method of teaching.”

\section{Acknowledgements}


The researchers would like to thank the members of the two MEST classes who volunteered their time to participate in this study. Further, they would like to extend thanks to their institutions, all colleges in the Higher Colleges of Technology system, for their support of this research initiative.

\section{References}

[1] Andrews, T. (November, 1999). Extending learning opportunities through technology - The video conference option. Paper presented at Effective courses / Effective Teaching at university conference, Women's College, The University of Queensland, Queensland, Australia.

[2] Andrews, T., \& Klease, G. (2002). Extending learning opportunities through a virtual faculty - the videoconference option. International Journal of Educational Technology, 3(1), 1-11.

[3] Butler, J., Hyde, S., \& Dougherty, P. (2010, December). [Focus group transcriptions: Innovations in postgraduate education]. Unpublished raw data.

[4] Dougherty, P., Butler, J., \& Hyde, S. (2010, December). The academic journey in the UAE: MEST student reflections. Retrieved February 15, 2011 from, Web site: <www.quia.com/ servlets/quia.activities.sv.summary.SummarySV?repo $\mathrm{rt}=$ Session\&activity_id=9692804>.

[5] Dziuban, C.D., Hartman, J.L., \& Moskal, P.D. (2004). Blended learning. EDUCAUSE, 2004 (7), 3.

[6] Eriks-Brophy, A., Quittenbaum, J., Anderson, D., Nelson, T. (2008) Part of the Problem or Part of the Solution? Communication Assessments of Aboriginal Children Residing in Remote Communities Using Videoconferencing. Clinical Linguistics \& Phonetics, 22 (8), 589-609.

[7] Exeter, U. o. (2008). Video conferencing for teaching and learning. Retrieved February 20, 2011 from, Web site: < as.exeter.ac.uk/support/ educationenhancementprojects/videoconferencing/>

[8] Gates Bill. Interview by Stephen Sackur. Hardtalk. BBC World News. BBC, London: 18 May . 2011. Television.

[9] George-Walker, L. \& Keeffe, M. (2004). Selfdetermined blended learning: a case study of blended learning design. Higher Education Research and Development, 29 (1), 1 - 19.

[10] Gill, D., Parker, C., \& Richardson, J. (2005). Twelve tips for teaching using videoconferencing. Medical Teacher, 27 (7), 573 - 577.
[11] Gillies, D. (2008). Student perspectives on videoconferencing in teacher education at a distance. Distance Education, 29 (1), 108 - 118.

[12] Hyde, S., Butler, J., \& Dougherty, P. (2010, December). [Panel discussion transcript: Innovations in postgraduate education]. Unpublished raw data.

[13] Lawson, T., Comber, C., Gage, J., \& CullumHanshaw, A. (2010). Images of the future for education? Videoconferencing: A literature review. Technology, Pedagogy and Education, 19 (3), 295 314.

[14] Luck, J. (2000). Building a Learning community Using Interactive Videoconferencing. Rockhampton: Central Queensland University.

[15] Motteram, G. (2006), 'Blended' education and the transformation of teachers: a long-term case study in postgraduate UK Higher Education. British Journal of Educational Technology, 37: 17-30. 